\title{
A paridade pode influenciar na alimentação do lactente nos primeiros seis meses de vida?
}

\author{
Can parity influence infant feeding in the first six months of life?
}

Renata Oliveira Neves (https://orcid.org/0000-0003-3064-4109) ${ }^{1}$

Juliana Rombaldi Bernardi (https://orcid.org/0000-0002-6803-4472) ${ }^{2}$

Clécio Homrich da Silva (https://orcid.org/0000-0003-2847-3961) ${ }^{2}$

Marcelo Zubaran Goldani (https://orcid.org/0000-0002-5302-284X) ${ }^{2}$

Vera Lucia Bosa (https://orcid.org/0000-0002-6283-9640) ${ }^{1}$

${ }^{1}$ Núcleo de Estudos em Saúde da Criança e do Adolescente, Universidade Federal do Rio Grande do Sul (UFRGS). R. Ramiro Barcelos 2400, Santa Cecília. 90035-003 Porto Alegre RS Brasil. renataoliveiraneves@ gmail.com

${ }^{2}$ Programa de PósGraduação em Saúde da Criança e do Adolescente, Departamento de Pediatria, Faculdade de Medicina, UFRGS. Porto Alegre RS Brasil.

\begin{abstract}
This article aims to evaluate the influence of parity on breastfeeding and introduction of complementary feeding in children up to six months after childbirth. Longitudinal study conducted through a convenience sample of mother-child pairs, selected at postpartum and accompanied until the sixth month of infant's life, between 2011 and 2016, in Porto Alegre, Brazil. There was an analysis of the time taken in the first feeding after birth, practice and time of breastfeeding, consumption of other types of milk and introduction of complementary feeding. The sample consisted of 161 dyads, with 74 primiparous and 87 multiparous. Multiparous women breastfed their babies sooner in the first 24 hours post-partum ( $p=0.019$ ). The offering of other kinds of milk showed no difference in relation to parity, as the moment of introduction to infant nutrition, although both primiparous and multiparous did it before the age of four months. Parity seemed to influence the timing of first breastfeeding offer, but not the introduction of complementary feeding, although this occurred in an early way. In this sense, it is necessary to disseminate more information about breastfeeding and infant feeding during prenatal and childcare, to improve maternal and child health.
\end{abstract}

Key words Parity, Postpartum period, Breast feeding, Infant nutrition
Resumo $O$ objetivo deste artigo é avaliar a influência da paridade no aleitamento materno e na introdução da alimentação complementar nos primeiros seis meses de vida. Estudo longitudinal com uma amostra de conveniência de pares mães-filhos, selecionados no pós-parto e acompanhados até o sexto mês de vida, de 2011 a 2016, em Porto Alegre, Brasil. Foi analisado o tempo da primeira mamada após o nascimento, a prática e o tempo de aleitamento materno, o consumo de outros leites e a introdução da alimentação complementar. A amostra consistiu de 161 díades, com 74 primíparas e 87 multíparas. As multíparas iniciaram antes o aleitamento materno nas primeiras 24 horas pós-parto $(p=0,019)$. A oferta de outros leites não mostrou diferença em relação à paridade, assim como o momento da introdução alimentar; ainda que tanto primíparas como multíparas o fizeram antes dos quatro meses de vida. A paridade pareceu influenciar o momento da primeira oferta do aleitamento materno, mas não a introdução da alimentação complementar, apesar de esta ter ocorrido precocemente. Neste sentido, se faz necessária a maior difusão de informações sobre aleitamento materno e alimentação infantil durante a assistência pré-natal e puericultura, para melhoria da saúde materno-infantil.

Palavras-chave Paridade, Período pós-parto, Aleitamento materno, Nutrição do lactente 


\section{Introdução}

A partir do nascimento, a amamentação é a principal escolha para a alimentação do lactente ${ }^{1}$, conferindo inúmeros benefícios tanto para a nutriz quanto para o lactente ${ }^{2}$.

Dentre os diversos fatores envolvidos na prática do aleitamento materno (AM), a paridade parece exercer influência, porém com poucos estudos publicados na literatura. Um deles verificou que as mães primíparas foram mais propensas a iniciar o AM, mas o mantiveram por menos tempo e introduziram prematuramente alimentos complementares. Uma hipótese é de que elas têm menor autoconfiança na habilidade de amamentar $^{3-5}$. Já a multiparidade mostrou-se como fator de proteção ao AM exclusivo, com as mães mantendo esta prática por um maior período de tempo ${ }^{6}$. Porém, conforme estudos anteriormente publicados, ainda existem controvérsias sobre a relação do número de partos da mãe com o AM e a influência da paridade sobre a introdução da $\mathrm{AC}$ ao longo do primeiro semestre de vida foi pouco investigada.

A respeito das recomendações, o leite materno (LM) deve ser ofertado de forma exclusiva até os seis meses de idade, sem oferecer água, chás ou qualquer outro alimento; e complementado até os dois anos ou mais ${ }^{7}$. Para as crianças amamentadas exclusivamente, a alimentação complementar (AC), a partir do sexto mês, deve ser introduzida de forma lenta e gradual, quando a mesma apresenta maturidade fisiológica e neurológica para receber outros alimentos, mantendo a prática do $\mathrm{LM}^{8}$. A AC deve ser adequada às necessidades nutricionais da criança, respeitando seus sinais de apetite e saciedade.

O período de introdução da AC é considerado de rápido crescimento e desenvolvimento infantil concomitante a grandes mudanças dietéticas, com exposição a novos alimentos, sabores e experiências alimentares 9 . A AC deve incluir os grupos alimentares de uma nutrição completa: grãos, raízes e tubérculos, leguminosas e oleaginosas, produtos lácteos, carnes e ovos, frutas e legumes ${ }^{10}$.

Quando comparada a introdução de AC entre lactentes que receberam LM e fórmula láctea, nos que receberam fórmula ela ocorre mais precocemente, entre três e cinco meses de idade. Além disto, puérperas que amamentam preferem oferecer aos seus filhos alimentos caseiros aos industrializados, enquanto que as que já utilizam fórmulas lácteas infantis tendem a introduzir outros alimentos industrializados na alimentação infantil ${ }^{11}$.
Considerando a relevância do tema exposto, este estudo investigou a influência da paridade na amamentação e na introdução de AC em lactentes ao longo dos seis primeiros meses de vida; verificando a diferença na prevalência de $\mathrm{AM}$ exclusivo, o momento da introdução de fórmula láctea infantil e comparando o momento e a forma da introdução da AC, entre puérperas primíparas e multíparas.

\section{Métodos}

Foi realizado um estudo longitudinal com uma amostra de pares mães-filhos, no período de 2011 a 2016. O estudo utilizou os dados de um projeto maior prospectivo controlado intitulado IVAPSA (Impacto das Variações do Ambiente Perinatal sobre a Saúde do Recém-Nascido nos Primeiros Seis Meses de Vida), cujo objetivo foi investigar os efeitos de diferentes ambientes intrauterinos e de condições perinatais sobre crescimento, neurodesenvolvimento, nutrição, comportamento e metabolismo da criança, e identificar seus efeitos deletérios ${ }^{12}$.

A coleta de dados foi realizada no Hospital de Clínicas de Porto Alegre (HCPA), no Hospital Fêmina e no Hospital Nossa Senhora da Conceição, ambos pertencentes ao Grupo Hospitalar Conceição (GHC).

Os critérios de inclusão foram: puérperas residentes no município de Porto Alegre, Rio Grande do Sul. Os critérios de exclusão foram: puérperas com teste positivo para HIV/AIDS, tabagistas ou que não conseguiram amamentar seus filhos no primeiro dia de vida e RN gemelares, pré-termos (idade gestacional inferior a 37 semanas), pequenos para a idade gestacional (PIG), com doenças congênitas ao nascimento, ou que necessitaram de internação hospitalar. Para este estudo, não incluímos as puérperas com doenças crônicas não transmissíveis.

O cálculo do tamanho da amostra do presente estudo foi baseado no estudo de DACHEW; BIFFTU $^{13}$, que objetivou investigar o acesso a práticas de aleitamento materno e fatores associados. Para este cálculo foi considerado poder de $80 \%$ e nível de significância de 5\%, com capacidade de detectar uma diferença de 1 mês entre as médias de $\mathrm{AM}$, considerando o desvio padrão de 1.7 comum aos grupos de paridade. Chegouse ao tamanho amostral mínimo de 94 sujeitos acrescentando $20 \%$ para possíveis perdas e recusas, totalizando 116 díades mãe-filho, igualmente distribuídas em relação à paridade. 
As seis entrevistas foram realizadas entre $24 \mathrm{e}$ 48 horas após o parto, aos sete e 15 dias e no primeiro, terceiro e sexto mês de vida da criança. Os entrevistadores foram treinados, periodicamente, a fim de padronizar as entrevistas e as técnicas de coleta. A seleção das puérperas, de modo aleatório, foi feita pela análise dos prontuários, nos alojamentos conjuntos dos hospitais, onde foi realizada a primeira entrevista. As demais ocorreram no domicílio ou no Centro de Pesquisa Clínica do HCPA, conforme disponibilidade das mães. Foram aplicados questionários sobre informações socioeconômicas maternas e familiares, obstétricas e sobre a alimentação da criança. Foi mantido anonimato dos participantes, com codificação dos dados e número de identificação.

Nas primeiras 48 horas após o parto, foi realizado o convite às puérperas para participarem da pesquisa, assim como a explicação dos seus objetivos. Mediante assinatura do Termo de Consentimento Livre e Esclarecido (TCLE), se procedia à primeira entrevista, por meio de um questionário aplicado pelo pesquisador com perguntas gerais sobre dados socioeconômicos e demográficos, sobre o parto e o RN, além de informações no prontuário médico hospitalar.

$\mathrm{Na}$ segunda entrevista, posteriormente à primeira semana após o parto, foram coletadas informações sobre a alimentação da criança na alta hospitalar e o tempo decorrido entre o parto e a primeira mamada. Nesta e nas entrevistas subsequentes, foi indagado à mãe se o seu filho mamava no peito (e se parou de mamar, com quantos dias), se já havia recebido outro leite, líquidos à base de água (chá, suco e refrigerante) ou alguns alimentos comumente oferecidos nesta faixa etária, contemplando todos os grupos alimentares.

As demais variáveis utilizadas foram: raça, idade e escolaridade materna, renda familiar mensal, presença de companheiro no domicílio, número de consultas pré-natal, Índice de Massa Corporal (IMC) pré-gestacional e tipo de parto. As variáveis relacionadas ao AM foram: tempo da primeira mamada após o nascimento; prática de AM e aleitamento materno exclusivo (AME); tempo de AM e AME e prática de consumo de fórmulas lácteas infantis caseiras, à base de leite de vaca, ou industrializadas.

A elaboração destas variáveis obedeceu aos conceitos de aleitamento materno segundo a Organização Mundial da Saúde (OMS $)^{10}$ : AM após o nascimento, que consistiu no tempo, em minutos, entre o nascimento da criança e a primeira mamada ao peito (foram excluídas do estudo seis mães que relataram ter amamentado seus filhos após o primeiro dia de vida); AM, quando a criança se encontrava em aleitamento materno, independente de outros líquidos ou alimentos oferecidos; AM exclusivo quando a criança recebia apenas LM, sem ser oferecido qualquer outro líquido ou sólido, com exceção de suplementos e medicamentos.

As variáveis relacionadas à $\mathrm{AC}$ foram agrupadas segundo a introdução dos seguintes grupos alimentares, propostos pelo Ministério da Saúde ${ }^{8}$ : cereais (inserção de cereais enriquecidos ou não no leite oferecido ou bolacha doce), verduras, frutas, leites e derivados (leite de vaca, iogurtes ou queijo), carnes (carnes, miúdos ou ovos), leguminosas, alimentos açucarados (suco artificial, refrigerante, açúcar adicional, achocolatado, mel, bolacha recheada ou wafer, chocolate, bombom, bala, pirulito, gelatina, pudim, sacolé, sorvete ou picolé) e outros (café, sopa industrializada, embutidos, salgadinho ou frituras). As categorias de introdução dos alimentos foram divididas nos tempos igual ou inferior a 4 meses e entre 5-6 meses de vida da criança. Excluiu-se das análises as crianças que não haviam consumido os alimentos citados.

As variáveis categóricas foram apresentadas por meio de número absoluto e percentual e as variáveis contínuas por média e desvio padrão $( \pm \mathrm{DP})$ ou mediana e intervalo interquartílico [percentil 25-75]. O teste qui-quadrado de Pearson ou exato de Fisher foi utilizado para detectar diferenças entre proporções e o teste $t$ de student ou Mann Whitney para detectar diferenças entre médias e medianas. Regressão Logística foi realizada para examinar a associação entre a paridade e a introdução da AC, ajustado para variáveis maternas (renda familiar, idade materna e IMC pré-gestacional). Para todas as análises, foi considerado nível de significância de 5\% $(\mathrm{p}<0,05)$ e intervalo de confiança de $95 \%$. Os dados foram processados e analisados pelo programa estatístico SPSS ${ }^{\circledR}$ (Statistical Package for the Social Sciences) versão 18.0 (SPSS Inc., Chicago, IL, EUA).

O estudo foi aprovado pelo Comitê de ética em Pesquisa (CEP) dos hospitais GHC e HCPA.

\section{Resultados}

Ao total, 209 díades foram recrutadas; dentre estas, houve 48 recusas, chegando-se à amostra final de 161 díades, contando com 74 primíparas $(45,96 \%)$ e 87 multíparas $(54,03 \%)$. A média (DP) do número de filhos foi 2,53 $( \pm 1,21)$.

A maioria das mulheres era da raça branca, com maior ocorrência de parto vaginal. Entre as 
primíparas encontraram-se as mulheres mais jovens $(\mathrm{p}<0,001)$, com maior renda familiar $(\mathrm{p}=$ $0,045)$ e maior número de consultas pré-natal ( $p$ $=0,048)$. A renda convertida para a média do salário mínimo (SM) nacional no período de 2011 a 2016 mostrou que as primíparas tinham uma renda de 0,3 SM maior. Além disto, estas iniciaram a gestação com menor valor de IMC $(\mathrm{p}=0,017)$ quando comparadas às multíparas (Tabela 1 ).

A prática de AM entre as mães primíparas e multíparas foi semelhante, tanto para AM exclusivo quanto para AM total. As multíparas iniciaram antes o aleitamento materno no pós-parto imediato $(p=0,019)$. O tempo médio de AM em suas categorias e a frequência da oferta de outros leites que não o materno, como fórmulas lácteas infantis industrializadas ou leite de vaca não mostrou diferença estatisticamente significativa $(\mathrm{p}=0,169)$ (Tabela 2).

Em relação à introdução alimentar, não houve diferença significativa entre mães primíparas e multíparas. Ambas ofereceram alimentos precocemente aos seus filhos, antes mesmo dos quatro meses de vida. (Tabela 3). A análise de Regressão Logística não demonstrou associação significativa entre a paridade e a introdução dos alimentos complementares (dados não mostrados), quando ajustados às covariáveis maternas (renda familiar, idade materna e IMC pré-gestacional).

\section{Discussão}

O objetivo do estudo foi avaliar a influência da paridade sobre a alimentação no primeiro semestre de vida do lactente e seus fatores associados; um tema ainda pouco estudado na literatura. Os resultados demonstraram que puérperas multíparas amamentaram mais prontamente o $\mathrm{RN}$ logo após o parto. Independente da paridade, houve oferta frequente de outros leites, introdução precoce da AC e semelhança nas prevalências de AM exclusivo e total.

Estudos anteriores observaram que, em crianças menores de um ano, a primiparidade mostrou-se como um fator de risco para o abandono do AM logo após o parto e para o desmame de AM total ${ }^{5,14}$. Nos lactentes de seis meses de vida ou com idade inferior, os riscos da amamentação não ser exclusiva são a baixa escolaridade materna, a primiparidade e o uso de chupeta. Ao contrário, a multiparidade é considerada fator de proteção para o desmame precoce ${ }^{6}$. Ainda assim, uma minoria de mães amamenta exclusivamente até o sexto mês de vida do lactente ${ }^{9}$.
A paridade também mostrou relação com a idade gestacional, o tipo de parto, o desejo de amamentar e com o início imediato da amamentação após o parto. Uma pesquisa prévia ${ }^{2}$ demonstrou que mulheres que realizaram parto cesárea eletiva tiveram maior planejamento para amamentação e iniciaram mais prontamente o AM quando comparadas à cesárea não-eletiva. Assim, 40\% destas puérperas se mostraram incapazes de amamentar seus filhos na primeira tentativa, comparado com $25 \%$ das que tiveram parto vaginal ou cesárea planejada. Tanto a cesárea planejada quanto a de emergência se caracterizaram como tendo maior propensão a suspender o aleitamento materno antes dos quatro meses de vida do lactente.

Analisando as covariáveis maternas, a média de idade das primíparas foi menor que a das multíparas. O mesmo resultado foi encontrado por Oliveira et al. ${ }^{3}$, que descreveram uma associação entre puérperas jovens e um menor tempo de AM. Os autores sugeriram que isto poderia estar relacionado com menor nível de escolaridade materna, menor poder aquisitivo e por união conjugal não estável. No presente estudo, diferentemente, não houve diferença significativa em relação ao estado conjugal; e as mães multíparas apresentaram menor renda do que as primíparas. Em relação à influência do poder econômico familiar, Victora et al..$^{15}$ demonstraram que as mulheres com menor poder aquisitivo tendem a amamentar por mais tempo que as de maior poder, especialmente nos países de renda média.

As mães primíparas apresentaram um maior número de consultas durante sua gestação. Este achado se mostrou consistente com a literatura, em que a multiparidade se relaciona ao atraso no início das consultas pré-natal, tendo como consequência o menor número de consultas durante a gestação ${ }^{16}$. O Caderno de Atenção Básica, número 23 do Ministério da Saúde ${ }^{17}$, ressalta a importância do acompanhamento pré-natal, independente da paridade.

Observa-se que outro desfecho importante relacionado à paridade é a obesidade. Pesquisa realizada em 2015 no Brasil $^{18}$ demonstrou que esta é mais prevalente entre as mulheres que já possuem filhos, aumentando exponencialmente em mulheres com mais de dois filhos. No presente estudo, estes achados foram confirmados, pois a maior mediana de IMC pré-gestacional foi observada em multíparas. Considerando a influência da obesidade sobre o AM, supõe-se que puérperas com alto IMC sofrem maior desconforto social por amamentar em público; sendo assim, a 
Tabela 1. Características demográficas e socioeconômicas da amostra (IVAPSA, Porto Alegre).

\begin{tabular}{|c|c|c|c|c|}
\hline $\begin{array}{c}\text { Características familiares e } \\
\text { maternas }\end{array}$ & Primíparas $(n=74)$ & Multíparas $(\mathrm{n}=87)$ & Total $(n=161)$ & $\mathbf{p}$ \\
\hline \multicolumn{5}{|l|}{ Cor da pele/raça, n (\%) } \\
\hline Branca & $49(66,2)$ & $49(56,3)$ & $98(60,9)$ & $0,263^{1}$ \\
\hline Não Branca & $25(33,8)$ & $38(43,7)$ & $63(39,1)$ & \\
\hline Idade materna (anos), média \pm DP & $23,67 \pm 6,80$ & $27,48 \pm 6,54$ & $25,73 \pm 6,91$ & $<0,001^{2}$ \\
\hline $\begin{array}{l}\text { Escolaridade materna (anos), } \\
\text { mediana [P25-P75] }\end{array}$ & $11,0[8,0-11,0]$ & $10,0[8,0-11,0]$ & $11,0[8,0-11,0]$ & $0,122^{3}$ \\
\hline $\begin{array}{l}\text { Renda familiar mensal (reais), } \\
\text { mediana }[\mathrm{P} 25-\mathrm{P} 75]^{\star}\end{array}$ & $2000[1500-3000]$ & $1800[1000-2650]$ & $2000[1210-3000]$ & $0,045^{3}$ \\
\hline \multicolumn{5}{|l|}{ Mora com companheiro, $\mathrm{n}(\%)$} \\
\hline Sim & $63(85,1)$ & $73(83,9)$ & $136(84,5)$ & $1,000^{1}$ \\
\hline Não & $11(14,9)$ & $14(16,1)$ & $25(15,5)$ & \\
\hline $\begin{array}{l}\text { Pré-natal (consultas), mediana } \\
{[\text { P25-P75] }}\end{array}$ & $9[7-10]$ & $8[6-10]$ & $8[6-10]$ & $0,048^{3}$ \\
\hline $\begin{array}{l}\text { IMC pré-gestacional }\left(\mathrm{Kg} / \mathrm{m}^{2}\right) \\
\text { mediana }[\mathrm{P} 25-\mathrm{P} 75]^{*}\end{array}$ & $22,06[19,98-26,13]$ & $24,46[21,92-27,41]$ & $23,63[20,81-26,99]$ & $0,017^{3}$ \\
\hline \multicolumn{5}{|l|}{ Tipo de parto, n (\%) } \\
\hline Vaginal & $51(68,9)$ & $63(72,4)$ & $114(70,8)$ & $0,755^{1}$ \\
\hline Cesárea & $23(31,1)$ & $24(27,6)$ & $47(29,2)$ & \\
\hline
\end{tabular}

duração da amamentação difere fortemente entre mulheres com diferentes $\mathrm{IMC}^{19}$. Ademais, estudo previamente publicado pelo grupo IVAPSA ${ }^{20} \mathrm{de}-$ monstrou que mães com sobrepeso ou obesidade pré-gestacional apresentaram iniciação tardia do aleitamento materno no pós-parto imediato.

A importância da amamentação logo após o parto já foi bem estabelecida. No presente estudo, o tempo médio se deu entre 30 e 60 minutos após o nascimento, acima da média brasileira. A prevalência de AM exclusivo antes dos seis meses de vida da criança na II Pesquisa de Prevalência de Aleitamento Materno nas Capitais Brasileiras e Distrito Federal ${ }^{21}$ foi $41,0 \%$. Na atual pesquisa, nenhuma criança chegou aos seis meses em AM exclusivo, o que pode ter ocorrido devido à grande oferta de fórmulas lácteas infantis e alimentos complementares. A duração mediana de AM total nas capitais foi de 54,1 dias, estando aquém da encontrada no atual estudo. Particularmente, a Região Sul do Brasil apresentou a maior prevalência de uso de chupetas pelas crianças, contribuindo assim para o menor tempo de AM. A crescente divulgação e comercialização de fórmulas lácteas também parecem influenciar a prevalência de AM. A indústria de substitutos do LM é grande e crescente, e sua propaganda enfraquece os esforços para melhorar a amamentação ${ }^{22}$.
A introdução muito precoce de água, chás, outros leites, comida salgada e frutas, sabidamente, influencia negativamente a amamentação e é muito utilizada na Região Sul do Brasil ${ }^{21}$. Estes dados corroboram com o presente estudo, em que mais da metade das puérperas entrevistadas no primeiro mês pós-parto, independente da paridade, já ofertavam outros alimentos diferentes do LM e, aos 4 meses de vida, havia grande consumo de vários grupos alimentares. É de se destacar que algumas puérperas entrevistadas não tinham a compreensão de que a oferta de outros líquidos não configura AM exclusivo. Estes resultados, como também os encontrados a nível nacional, demonstram que as metas propostas pelo $\mathrm{MS}^{17}$ e pela Organização Mundial da Saúde (OMS) ${ }^{23}$ ainda estão muito distantes de serem cumpridas.

Em relação à AC, no presente estudo, tanto as mães primíparas quanto as multíparas ofereceram alimentos às crianças de forma precoce, antes mesmo dos quatro meses de vida. A regressão logística multivariada, ajustada às covariáveis maternas, não demonstrou relação entre paridade e AC precoce, o que pode ter ocorrido devido ao pequeno número da amostra. Corroborando com estes achados, uma pesquisa realizada recentemente no Paraná, Curitiba, com 80 crianças 
Tabela 2. Tempo de aleitamento materno e consumo de fórmula infantil entre primíparas e multíparas (IVAPSA, Porto Alegre).

\begin{tabular}{lccc}
\hline \multicolumn{1}{c}{$\begin{array}{c}\text { Aleitamento } \\
\text { materno }\end{array}$} & Primíparas & Multíparas & \\
\cline { 2 - 3 }$n$ & $\mathbf{n}(\%)^{\star}$ & $\mathbf{n}(\%)^{\star}$ & \\
\hline AM depois do & $60[30-120]$ & $30[15-120]$ & $0,019^{1}$ \\
nascimento & & & \\
$($ min $)$, mediana & & & \\
{$[\mathrm{P} 25-\mathrm{P} 75], \mathrm{n}=141$} & & & \\
AM total & & &
\end{tabular}

7 dias $(n=1$

$\begin{array}{lrrr}\text { Sim } & 52(100,0) & 62(98,4) & 1,000^{3} \\ \text { Não } & 0(0) & 1(1,6) & \\ 5 \text { dias }(\mathrm{n}=102) & & \end{array}$

$\begin{array}{lrrr}\text { Sim } & 48(98,0) & 52(98,1) & 1,000^{3} \\ \text { Não } & 1(2,0) & 1(1,9) & \\ 1 \text { mês }(\mathrm{n}=110) & & & \\ \quad \text { Sim } & 46(97,9) & 60(95,2) & 0,634^{3} \\ \text { Não } & 1(2,1) & 3(4,8) & \\ 3 \text { meses (n=107) } & & & \\ \quad \text { Sim } & 43(87,8) & 50(86,2) & 1,000^{2} \\ \text { Não } & 6(12,2) & 8(13,8) & \\ 6 \text { meses (n=94) } & & & \\ \quad \text { Sim } & 30(65,2) & 35(72,9) & 0,559^{2} \\ \text { Não } & 16(34,8) & 13(27,1) & \end{array}$

\section{AM exclusivo}

7 dias $(n=114)$

\begin{tabular}{lrrr} 
Sim & $41(78,8)$ & $47(75,8)$ & $0,872^{2}$ \\
Não & $11(21,2)$ & $15(24,2)$ & \\
15 dias (n=102) & & & \\
Sim & $33(67,3)$ & $29(54,7)$ & $0,270^{2}$ \\
Não & $16(32,7)$ & $24(45,3)$ & \\
1 mês (n=110) & & & \\
Sim & $19(40,4)$ & $19(30,2)$ & $0,359^{2}$ \\
Não & $28(59,6)$ & $44(69,8)$ & \\
3 meses (n=107) & & & \\
Sim & $15(30,6)$ & $10(17,2)$ & $0,162^{2}$ \\
Não & $34(69,4)$ & $48(82,8)$ & \\
6 meses (n=94) & & & \\
Sim & $0(0,0)$ & $0(0,0)$ & - \\
Não & $46(100,0)$ & $48(100,0)$ & \\
Tempo de AM & $150[60-$ & $150[90-$ & $0,637^{1}$ \\
total (dias), & $180]$ & $180]$ & \\
mediana & \multicolumn{3}{l}{}
\end{tabular}

mediana

[P25-P75], $\mathrm{n}=136$

Tempo de

AM exclusivo

(dias), mediana

$15[7-90] \quad 11[7-30] \quad 0,422^{1}$

[P25-P75], $\mathrm{n}=121$

IVAPSA: Impacto das Variações do Ambiente Perinatal sobre a Saúde do Recém-Nascido nos Primeiros Seis Meses de Vida; DP: desvio padrão; AM: aleitamento materno. Testes estatísticos: ${ }^{1}$ qui-quadrado; ${ }^{2}$ Mann Whitney; ${ }^{3}$ Exato de Fisher. ${ }^{\star} \mathrm{O}$ n de cada variável variou conforme as respostas válidas e as entrevistas de seguimento realizadas.

Tabela 3. Alimentação complementar entre primíparas e multíparas (IVAPSA, Porto Alegre).

\begin{tabular}{lrrr}
\hline \multirow{2}{*}{$\begin{array}{c}\text { Alimentação } \\
\text { complementar } \\
(\mathbf{n}=116)\end{array}$} & Primíparas & Multíparas & \\
Cereais & $\mathbf{n}(\%)^{*}$ & $\mathbf{n}(\%)^{*}$ & $\mathbf{p}$ \\
$\quad$ Nunca & $13(25,0)$ & $16(25,0)$ & $0,497^{1}$ \\
$\leq 4$ meses & $13(25,0)$ & $22(34,4)$ & \\
5-6 meses & $26(50,0)$ & $26(40,6)$ & \\
Verduras & & & \\
Nunca & $17(32,7)$ & $29(45,3)$ & $0,372^{1}$ \\
$\leq 4$ meses & $10(19,2)$ & $11(17,2)$ & \\
5-6 meses & $25(48,1)$ & $24(37,5)$ & \\
Frutas & & & \\
Nunca & $5(9,6)$ & $15(23,4)$ & $0,084^{1}$ \\
$\leq 4$ meses & $18(34,6)$ & $24(37,5)$ & \\
5-6 meses & $29(55,8)$ & $25(39,1)$ &
\end{tabular}

Leites e

derivados

Nunca

$\leq 4$ meses

$20(38,5)$

$23(35,9)$

$0,566^{1}$

5-6 meses

$21(40,4) \quad 22(34,4)$

$11(21,2) \quad 25(39,1)$

Carnes

Nunca

$\leq 4$ meses

$22(42,3)$

$33(51,6)$

$0,382^{1}$

5-6 meses

$9(17,3)$

$6(9,4)$

$21(40,4) \quad 25(39,1)$

Leguminosas

Nunca

$\leq 4$ meses

$20(38,5)$

$34(53,1)$

$6(9,4)$

5-6 meses

$6(11,5)$

$24(37,5)$

Alimentos

açucarados

Nunca

$\leq 4$ meses

$15(28,8)$

$19(29,7)$

$0,937^{1}$

5-6 meses

$25(48,1)$

$32(50,0)$

$12(23,1)$

$13(20,3)$

Outros

\begin{tabular}{lrrr} 
Nunca & $38(73,1)$ & $52(81,3)$ & $0,572^{1}$ \\
$\leq 4$ meses & $5(9,6)$ & $4(6,3)$ & \\
5-6 meses & $9(17,3)$ & $8(12,5)$ & \\
\hline
\end{tabular}

IVAPSA: Impacto das Variações do Ambiente Perinatal sobre a Saúde do Recém-Nascido nos Primeiros Seis Meses de Vida. Testes estatísticos: ${ }^{1}$ qui-quadrado.

entre seis e 24 meses, encontrou que para $35 \%$ das crianças, a AC ocorreu antes dos seis meses de vida, sendo que $16 \%$ destas receberam leite de vaca; e apenas $13,7 \%$ das crianças estavam em aleitamento materno exclusivo aos seis meses. A papa principal foi iniciada aos 5-6 meses, sendo a carne implementada apenas aos nove meses. Isto demonstrou que as mães ainda não têm conheci- 
mento de como e quando a alimentação alimentar deve ser iniciada ${ }^{24}$.

$\mathrm{Na}$ Etiópia, quando observadas 422 mães multíparas, os alimentos mais ofertados na introdução da $\mathrm{AC}$ foram líquidos à base de cereais infantis, seguido de leite de vaca, fórmula láctea infantil e comida da família ${ }^{25}$. Este mesmo estudo mostrou que as multíparas com maior nível educacional ofereceram a AC no momento adequado, o que pode ser explicado pela sua maior compreensão das demandas e dos benefícios desta introdução a partir dos seis meses, assim como uma capacidade de resistir às pressões e interferências externas na introdução de alimentos mais precocemente.

Entre as limitações do presente estudo podem ser citadas a seleção da amostra ter ocorrido apenas em hospitais públicos que prestam assistência pelo Sistema Único de Saúde (SUS), o seguimento das duplas mãe-filho somente até o sexto mês, e, portanto, não tendo informações sobre o AM e a AC até o final do primeiro ano de vida. A prevalência do AM e do momento da introdução da AC e sua qualidade podem não ser representativos da população em geral, mas sim da população da qual a amostra foi extraída.

Como pontos fortes destacam-se a qualidade da equipe de trabalho para coleta e análise dos dados e o emprego de questionários que permitiram a caracterização detalhada da amostra, um aspecto fundamental para a identificação de fatores que possam interferir na alimentação dos lactentes menores de seis meses. Ademais, dos seis momentos das entrevistas e avaliação, quatro ocorreram no primeiro mês de vida, possibilitando identificar possíveis variações na alimentação em um período crítico e precoce e, portanto, propício para intervenção.

\section{Conclusão}

A paridade materna exerceu influência no momento da primeira oferta de aleitamento materno logo após o parto. As mães multíparas parecem ter maior facilidade no manejo da amamentação, mas não apresentam diferenças na oferta de alimentação complementar quando comparadas com as primíparas. Faz-se necessário maior atenção dos profissionais de saúde sobre o aleitamento materno e a introdução da alimentação complementar na assistência pré-natal e durante a puericultura, para que as puérperas saibam ofertar aos seus filhos uma alimentação infantil de qualidade e no momento adequado. Desta forma, juntamente com a consolidação das políticas públicas na área da saúde materno-infantil, será possível prevenir as doenças crônicas não transmissíveis no país. Os autores declararam não haver conflito de interesses.

\section{Colaboradores}

MZ Goldani, CH Silva e VL Bosa trabalharam na concepção da pesquisa. RO Neves e JR Bernardi trabalharam na coleta de dados. RO Neves trabalhou na redação do artigo. CH Silva, VL Bosa e JR Bernardi trabalharam na revisão do artigo. 


\section{Referências}

1. Vasconcelos MGL, Lira PIC, Lima, MC. Duração e fatores associados ao aleitamento materno em crianças menores de 24 meses de idade no estado de Pernambuco. Rev Bras Saude Mater Infant 2006; 6(1):99-105.

2. Hobbs AJ, Mannion CA, McDonald SW, Brockway M, Tough SC. The impact of caesarean section on breastfeeding initiation, duration and difficulties in the first four months postpartum. BMC Pregnancy Childbirth 2016; 16:90.

3. Oliveira JS, Joventino ES, Dodt RCM, Veras JEGLF, Ximenes LB. Fatores associados ao desmame precoce entre multíparas. Revista da Rede de Enfermagem do Nordeste. Rev Rene 2010; 11(4):95-102.

4. Lee, HMH, Durham J, Booth J, Sychareun V. A qualitative study on the breastfeeding experiences of firsttime mothers in Vientiane, Lao PDR. BMC Pregnancy Childbirth 2013; 13:223.

5. Oribe M, Lertxundi A, Basterrechea M, Begiristain H, Marina LS, Villar M, Dorronsoro M, Amiano P, Ibarluzea J. Prevalence of factors associated with the duration of exclusive breastfeeding during the first 6 months of life in the INMA birth cohort in Gipuzkoa. Gac Sanit 2015; 29(1):4-9.

6. Salustiano LPQ, Diniz ALD, Abdallah VOS, Pinto RMC. Fatores associados à duração do aleitamento materno em crianças menores de seis meses. Rev Bras Ginecol Obstet 2012; 34(1):28-33.

7. Brasil. Ministério da Saúde (MS). Dez passos para uma alimentação saudável para crianças brasileiras menores de dois anos. Brasília: MS; 2010.

8. Brasil Ministério da Saúde (MS). Dez passos para uma alimentação saudável - Guia alimentar para crianças menores de dois anos. Brasília: MS; 2013.

9. Fewtrell M, Bronsky J, Campoy C, Domellöf M, Embleton N, Mis NF, Hojsak I, Hulst JM, Indrio F, Lapillonne A, Molgaard C. Complementary feeding: a position paper by the European Society for Paediatric Gastroenterology, Hepatology, and Nutrition (ESPGHAN) Committee on Nutrition. J Pediatr Gastroenterol Nutr 2017; 64(1):119-132.

10. World Health Organization (WHO). Indicators for assessing infant and young child feeding practices. Genebra: WHO; 2008.

11. Schiess S, Grote V, Scaglioni S, Luque V, Martin F, Stolarczyk A, Vecchi F, Koletzko B, European Childhood Obesity Project. Introduction of Complementary Feeding in 5 European Countries. J Pediatr Gastroenterol Nutr 2010; 50(1):92-98.

12. Bernardi, JR, Ferreira CF, Nunes M, Silva CH, Bosa VL, Silveira PP, Goldani MZ. Impact of perinatal different intrauterine environments on child growth and development in the first six months of life - IVAPSA birth cohort: rationale, design, and methods. BMC Pregnancy Childbirth 2012; 12:25.

13. Dachew BA, Bifftu BB. Breastfeeding practice and associated factors among female nurses and midwives at North Gondar Zone, Northwest Ethiopia: a cross-sectional institution based study. Int Breastfeed J 2014; 9:11.

14. França GVA, Brunken GS, Silva SM, Escuder MM, Venancio SI. Determinantes da amamentação no primeiro ano de vida em Cuiabá, Mato Grosso. Rev Saude Publica 2007; 41(5):711-718.
15. Victora CG, Bahl R, Barros AJD, França GVA, Horton S, Krasevec J, Murch S, Sankar MJ, Walker N, Rollins NC, Lancet Breastfeeding Series Group. Breastfeeding in the 21st century: epidemiology, mechanisms, and lifelong effect. Lancet 2016; 387(10017):475-490.

16. Aung TZ, Oo WM, Khaing W, Lwin N, Dar HT. Late initiation of antenatal care and its determinants: a hospital based cross-sectional study. Int J Community Med Public Health 2016; 3(4):900-905.

17. Brasil. Ministério da Saúde (MS). Caderno de Atenção Básica, número 23. Brasília: MS; 2015.

18. Ferreira RAB, Benício MHDA. Obesidade em mulheres brasileiras: associação com paridade e nível socioeconômico. Rev Panam Salud Publica 2015; 37(4/5):337-342.

19. Newby RM, Davies PSW. Antenatal breastfeeding intention, confidence and comfort in obese and nonobese primiparous Australian women: associations with breastfeeding duration. Eur J Clin Nutr 2016; 70(8):935-940.

20. Pinheiro TV, Goldani MZ, grupo IVAPSA. Maternal pre-pregnancy overweight/obesity and gestational diabetes interaction on delayed breastfeeding initiation. PLoS One 2018; 13(6):e0194879.

21. Brasil. Ministério da Saúde (MS). II Pesquisa de Prevalência de Aleitamento Materno nas Capitais Brasileiras e Distrito Federal. Brasília: MS; 2009.

22. Rollins NC, Bhandari N, Hajeebhoy N, Horton S, Lutter CK, Martines JC, Piwoz EG, Richter LM, Victora CG, Lancet Breastfeeding Series Group. Why invest, and what it will take to improve breastfeeding practices? Lancet 2016; 387(10017):491-504.

23. World Health Oeganization (WHO). The optimal duration of exclusive breastfeeding. Genebra: WHO; 2001.

24. Gurmini J, Porello EB, Belleza MSS, Silva KN, Kusma SZ. Análise da Alimentação Complementar em Crianças Entre 0 e 2 Anos de Escolas Públicas. Rev Med UFPR 2017; 4(2):55-60.

25. Shumey A, Demissie M, Berhane Y. Timely initiation of complementary feeding and associated factors among children aged 6 to 12 months in Northern Ethiopia: an institution-based cross-sectional study. BMC Public Health 2013; 13:1050.

Artigo apresentado em 09/10/2018

Aprovado em 01/02/2019

Versão final apresentada em 03/02/2019 